\title{
Primary Cutaneous Mucinous Carcinoma with Extramammary Paget's Disease: Eccrine or Apocrine?
}

\author{
Sun-Ju Oh · Young-Ok Kim \\ Department of Pathology, Kosin University \\ Gospel Hospital, Busan, Korea \\ Received: October 26, 2017 \\ Revised: November 17, 2017 \\ Accepted: November 21, 2017

\section{Corresponding Author} \\ Sun-Ju Oh, MD \\ Department of Pathology, Kosin University Gospel \\ Hospital, 262 Gamcheon-ro, Seo-gu, Busan 49267, \\ Korea \\ Tel: +82-51-990-6744 \\ Fax: +82-51-990 3080 \\ E-mail: 10highpowerfield@gmail.com
}

\begin{abstract}
Primary cutaneous mucinous carcinoma (PCMC) is an uncommon tumor of the sweat gland origin. The occurrence of PCMC is mostly in middle-aged and older patients, with a slight male predominance. Most cases of PCMC arise on the head, with a preference for eyelids. The histogenesis of PCMC, whether eccrine or apocrine, remains controversial. We report a rare case of PCMC with secondary extramammary Paget's disease in the groin of a 75-year-old man, which favored an apocrine origin. Furthermore, based on a review of the literature, we provide several histologic clues that can be used to differentiate PCMC from metastatic mucinous carcinoma.
\end{abstract}

Key Words: Cutaneous mucinous carcinoma; Skin adnexal tumor; Apocrine carcinoma; Paget's disease, extramammary
Primary cutaneous mucinous carcinoma (PCMC) is a rare sweat gland tumor, ${ }^{1}$ whereas the majority of mucinous carcinoma of the skin is metastatic. The common metastasis originates from the gastrointestinal tract and other sites such as the breast, salivary glands, lacrimal glands, nose and paranasal sinuses, bronchi, renal pelvis, and ovary. ${ }^{2}$ It occurs mostly in middleaged and older patients, with a slight male predominance. The histogenesis of PCMC, whether eccrine or apocrine, remains controversial. Several studies have suggested that the neoplasm showed eccrine differentiation, ${ }^{3-6}$ while others favored apocrine origin. $^{7-10}$

We report a case of PCMC with extramammary Paget's disease (EMPD), exhibiting the biphasic appearance of pure mucinous carcinoma and poorly differentiated carcinoma that favors apocrine differentiation. In addition, we discuss features that differentiate $\mathrm{PCMC}$ and metastatic mucinous carcinoma.

\section{CASE REPORT}

A 75-year-old man presented with recurrent pruritic eczema of the penis and scrotum that was refractory to medication of the local dermatologic clinic for one and a half years. The lesion consisted of an erythematous and indurated plaque with ulcer- ation and exudation (Fig. 1A). Review of the patient's history revealed that he underwent right hemicolectomy for adenocarcinoma in the ascending colon when he was 62 years old. He remained under regular follow-up for 13 years, during which no metastases were reported. Examination of skin biopsy from the inguinal folds revealed large cells with clear to eosinophilic cytoplasm that had infiltrated into the epidermis, singly and in clusters (Fig. 1B). The initial histologic diagnosis of the skin biopsy was EMPD. The preoperative instrumental investigations showed no evidence of recurrence on the previous surgical site or other underlying internal malignancy. The patient underwent a mapping biopsy at multiple sites to accurately establish the boundary of the lesion, followed by wide excision and closure of the defect with a skin graft.

The resected perineum and penile shaft showed ill-defined indurated nodules with a firm consistency. The lesion in the overlying epidermis measured $6 \mathrm{~cm}$, with a 1.5 -cm-sized mass in the dermis. The cut surface was solid and gray-white with focal mucinous appearance. On histologic examination, the lowpower view showed a relatively well-defined mass occupying the dermis and subcutis (Fig. 2B). The overlying epidermis showed irregular epidermal hyperplasia containing scattered large cells arranged singly or in groups, as in the previous skin biopsy, 
suggesting an epidermal pagetoid spread of underlying tumor cells (Fig. 1B). The tumor showed two composite features in which solid nests of poorly differentiated carcinoma were juxtaposed with paucicellular mucinous lakes with floating tumor cell clusters (Fig. 2C). The latter accounted for the majority of the tumor, about $70 \%$, and the rest consisted of poorly differentiated components. Tumor cells in both the mucinous pool and more cellular area revealed the same histological features of abundant eosinophilic cytoplasm and large vesicular nuclei with frequent mitoses up to 7/10 high power field (Fig. 2C, D). In
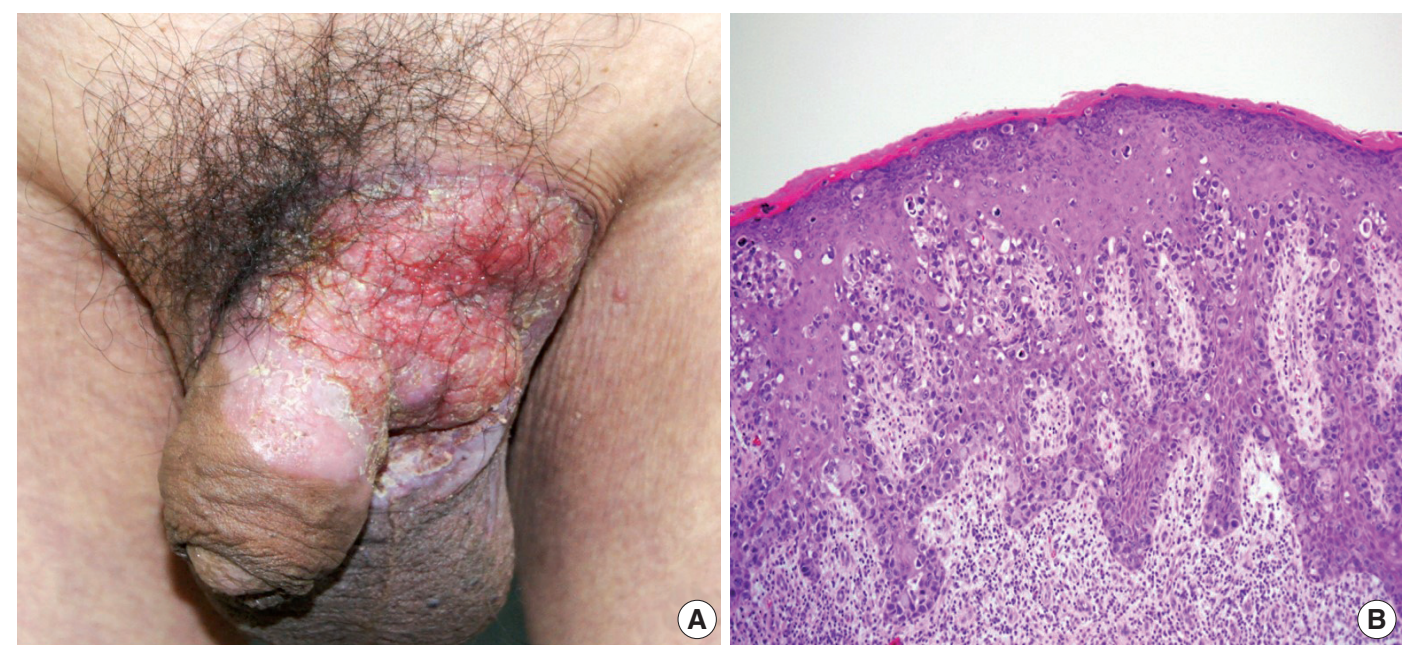

Fig. 1. (A) Erythematous plaque of the left groin with extension to the scrotum and penis. (B) Histology of preoperative skin biopsy revealing extensive involvement of the epidermis by large pagetoid cells with clear or eosinophilic cytoplasm.
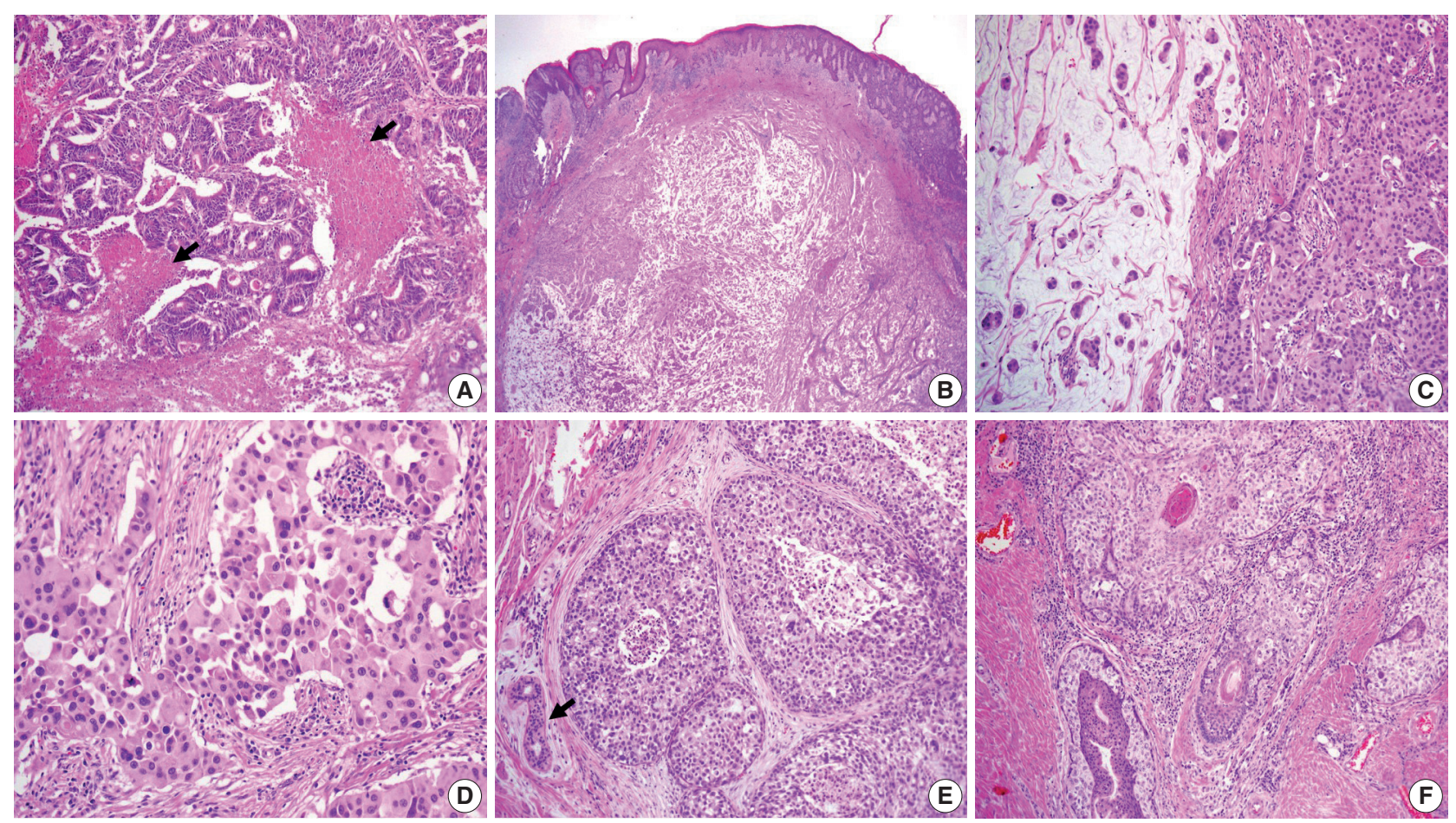

Fig. 2. (A) Moderately differentiated adenocarcinoma of the colon with dirty necrosis (arrows) diagnosed 13 years earlier. (B) Low magnification of the mass in the dermis with epidermal acanthosis due to pagetoid spread of the tumor cells. (C) Mucinous lakes with free-floating tumor nests (left) are juxtaposed with a cellular apocrine carcinoma (right), of which high magnification (D) reveals solid nests of tumor cells with round nuclei and abundant eosinophilic cytoplasm. (E) Comedo-type in situ component is depicted with normal apocrine glands (arrow). (F) Colonization of tumor cells in the hair follicle. 
situ lesions similar to ductal carcinoma in situ of the breast were also observed (Fig. 2E) with colonization of the hair follicles (Fig. 2F). Overall histology suggested a mucinous carcinoma with minor poorly differentiated components.

Histological differential diagnosis included PCMC and late metastasis of the previously diagnosed colon cancer. Histologic review of the patient's previous colon cancer showed a $4.3-\mathrm{cm}$ moderately differentiated adenocarcinoma with the assigned stage of T3N0M0. The tumor consisted of well-formed or cribriform patterns of tumor glands with frequently observed dirty necrosis (Fig. 2A). There was no mucin production observed in the skin lesion.

Immunohistochemical studies were performed. The Paget's and dermal tumor cells of the skin lesion were positive for cytokeratin (CK) 7, CK20, gross cystic disease fluid protein-15 (GCDFP-15) (Fig. 3A-C), and carcinoembryonic antigen (CEA), but negative for caudal-related homeobox gene 2 (CDX-2). On the other hand, tumor cells of the colon cancer were positive for CK20, CDX-2, CEA, but not gross cystic disease fluid protein 15 or CK7. Additional staining of human epidermal growth factor receptor 2 and estrogen receptor (ER) was observed in the skin lesion (Fig. 3D, E), but the lesion was negative for progesterone receptor (PR). Myoepithelial cells surrounding the carci- noma in situ were highlighted by CK5/6 immunostaining (Fig. 3F). The overall immunohistochemical findings of the skin lesion were different from those of colon cancer but similar to those of breast cancer, but similar to those of breast cancer, indicating sweat gland origin. On the basis of these histologic and immunohistochemical findings, the two malignancies were considered to differ in origin, and the present case was a primary mucinous carcinoma of the skin.

Another challenge was to determine whether the minor poorly differentiated components were eccrine or apocrine in origin because there was no clear distinction in histologic and immunohistochemical features. We concluded apocrine differentiation of the lesion due to the location in the groin, where apocrine glands are many in number, and to the resemblance of apocrine carcinoma in the breast even though decapitation secretion was absent. In addition, close approximation and colonization of the hair follicle (Fig. 2F) favored the origin of the follicularapocrine unit.

The final pathologic diagnosis was PCMC with EMPD showing apocrine differentiation.

This case was approved by the institutional review board (IRB) of Kosin University Gospel Hospital, and informed consent was waived by the IRB (IRB reference number: 2017-10-010).
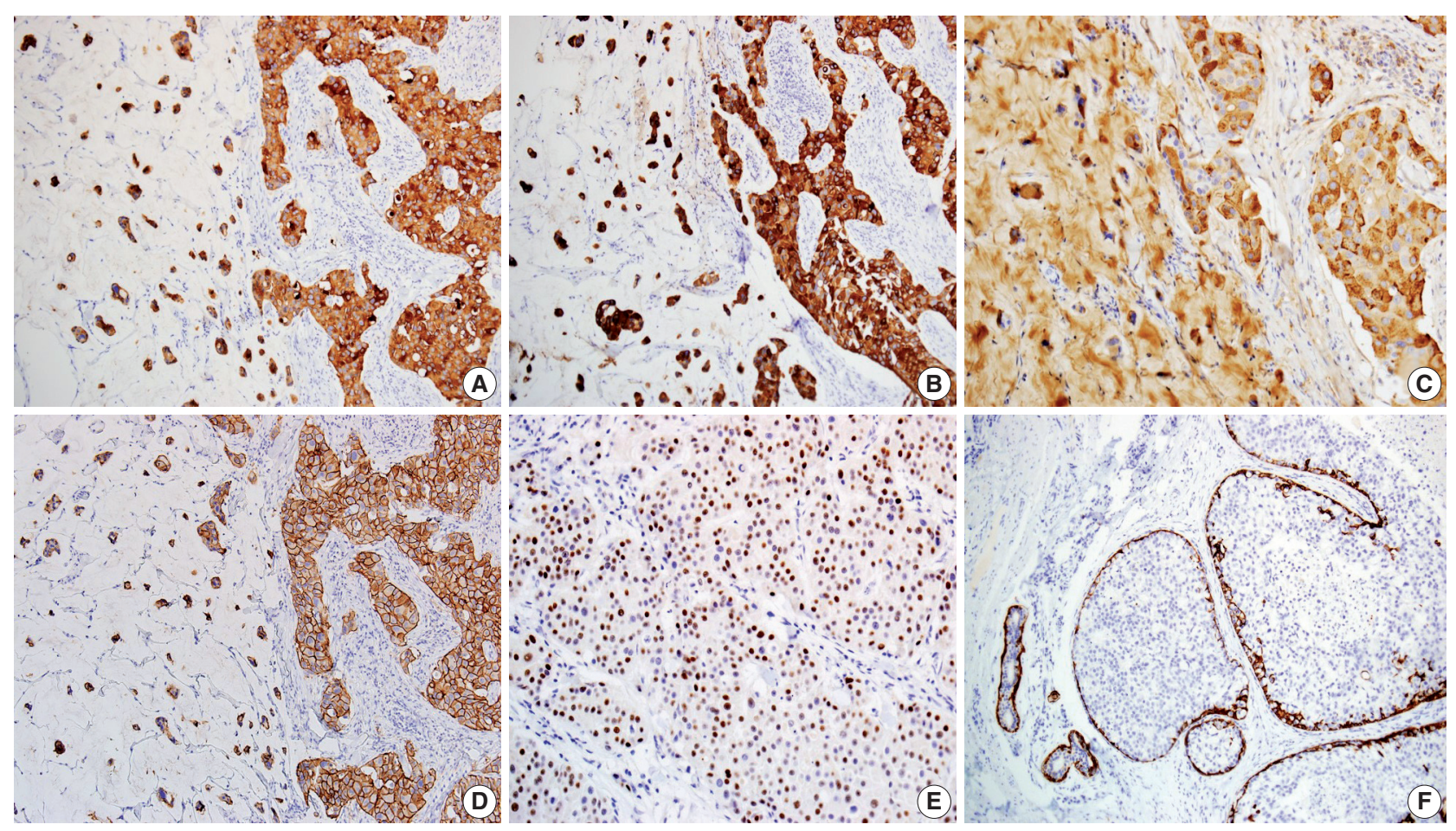

Fig. 3. Immunohistochemical findings of tumor cells showing positivity for cytokeratin (CK) 7 (A), CK20 (B), Gross cystic disease fluid protein 15 (C), human epidermal growth factor receptor 2 (D), and estrogen receptor (E). Myoepithelial cells of in situ components and normal apocrine glands highlighted by immunostaining for CK5/6 (F). 


\section{DISCUSSION}

PCMC is an uncommon subtype of sweat gland tumor. Only about 100 cases of PCMC have been reported ${ }^{1,6}$ since the first report by Lennox et al. ${ }^{11}$ in 1952. PCMC shows a slight male predominance and typically affects people aged $50-70$ years. The eyelid was most commonly affected, followed by the scalp, face, axilla, chest/abdominal wall, vulva, neck, extremity, canthus, groin, and ear. ${ }^{6}$ The primary lesion of PCMC is often solitary, and the size of the neoplasm varies in diameter from about $0.7-$ $8.0 \mathrm{~cm}$.

It is still controversial whether PCMC has eccrine or apocrine differentiation. Determining the eccrine or apocrine differentiation continues to be problematic because there is no clear distinction in histologic and immunohistochemical features. Robson et al..$^{12}$ suggested that apocrine differentiation was determined when encountering the tumor in its usual anatomic location with numerous apocrine glands and with its propensity for coexistence with follicular tumors, which means it is derived from the follicular-apocrine-sebaceous rather than eccrine axis. The presence of decapitation secretion is also known to be a hallmark of apocrine differentiation. However, some authors ${ }^{12}$ described apocrine carcinoma without decapitation secretion, and they relied on the identification of tumor cells with abundant eosinophilic cytoplasm in tumors that arises from a typical location including the axilla or groin, as in the present case.

For PCMC, some authors have favored eccrine differentiation based on immunohistochemical studies and ultrastructural analysis. ${ }^{3-5}$ On the other hand, other studies have suggested that PCMC has an apocrine origin. ${ }^{7-10}$ Riquena $e t$ al. ${ }^{7}$ suggested an apocrine origin for PCMC because it shows identical histologic features with mucinous carcinoma of the mammary glands, known as modified apocrine glands. Another study reported a case of PCMC coexisting with trichofolliculoma, also providing evidence of apocrine lineage. ${ }^{9}$

The present case revealed EMPD associated with underlying PCMC. EMPD is considered to be an intraepithelial adenocarcinoma typically involving vulvar, perianal, perineal, scrotal, and penile regions. ${ }^{13}$ EMPD sometimes occurs secondarily in association with cutaneous adnexal tumors or with internal malignancies. Some authors have shown that vulvar EMPD was associated with adnexal adenocarcinoma in $4 \%$ of cases and with a distant malignancy in $20 \%$, while perianal EMPD was associated with adnexal adenocarcinoma in $7 \%$ of cases and an internal malignancy in $14 \%$ of cases. ${ }^{14}$ There have been rare cases of PCMC coexisting with overlying EMPD, as in our case. ${ }^{15-17}$ Hurt et al. ${ }^{15}$ reported two cases of anogenital PCMC with EMPD, describing fibroepithelioma-like change of the overlying epidermis. The authors suggested that the anogenital mucinous carcinoma was associated with the EMPD, which had never been described in extraperineal mucinous carcinoma. To our knowledge, there have been no reports of EMPD coexisting with eccrine carcinoma in the groin. Instead, four cases of apocrine carcinoma with EMPD in this region have been reported in the English literature, ${ }^{14,18-20}$ which supports apocrine differentiation.

Another challenge in establishing the diagnosis of PCMC is the exclusion of metastasis from an underlying neoplasm because the majority of mucinous carcinomas in the skin are metastatic. The common metastasis is derived from the gastrointestinal tract, and other sites including the breast, salivary glands, lacrimal glands, nose and paranasal sinuses, bronchi, renal pelvis, and ovary have been reported. ${ }^{2}$ There are several histologic criteria and immunohistochemical patterns that can differentiate primary and secondary mucinous carcinomas. ${ }^{1,2,7}$ Riquena et al. ${ }^{7}$ suggested that histologic features such as larger clusters of cohesive neoplastic cells, fewer quantities of mucin, predominance of epithelium over mucin, and the absence of delicate fibrous septa favor metastasis. There have been other reports ${ }^{1,2}$ that the presence of an in situ component is most useful to establish PCMC especially when discriminating it from cutaneous metastasis of breast mucinous carcinoma. Morphologically, cutaneous and mammary lesions are indistinguishable and have immunophenotypic similarity, both expressing CK7, CEA, GCDFP 15, S-100 protein, ER, and PR. Qureshi et al. ${ }^{2}$ emphasized in situ components assessed by the presence of a myoepithelial cell layer identified by immunohistochemical staining for p63, CK5/6, calponin, and smooth muscle actin. The presence of an in situ component defines the neoplasm as primary cutaneous, but its absence does not exclude the diagnosis. A full clinical investigation is required to exclude metastasis and establish the origin. It is less challenging to exclude a metastasis of colorectal origin. Kazakov et al. ${ }^{1}$ suggested that dirty necrosis, frequently encountered in colorectal metastasis to the ovaries, is a good criterion of cutaneous mucinous carcinoma of intestinal origin. Tumor cells from colorectal adenocarcinoma were CK7-/CK20+ and invariably positive for CDX-2.

The current treatment for PCMC is excision with an at least 1-cm margin, as it is found to be resistant to chemotherapy and radiation. ${ }^{6}$ Prognosis is relatively good after complete surgical excision, with rare metastasis to regional lymph nodes. ${ }^{2}$ Prophylactic lymph node dissection should be considered in tumors of the axilla, as these have a higher rate of metastasis. ${ }^{5}$ Close follow- 
up is warranted as local recurrence is frequent at $29.4 \%$ in spite of a low metastasis rate and an indolent course. The overall mortality rate is $2 \%{ }^{6}$

In summary, PCMC is an uncommon variant of sweat gland tumor. Our case is a rare PCMC with EMPD. Minor poorly differentiated components were also observed, which suggested apocrine differentiation. However, further studies are required to clarify the histogenesis of PCMC. Clinical differentiation from metastatic mucinous carcinoma is most important, and a careful search for an in situ component can be a helpful morphologic finding that identifies the primary nature of the carcinoma in addition to the immunohistochemical panel.

\section{Conflicts of Interest}

No potential conflict of interest relevant to this article was reported.

\section{REFERENCES}

1. Kazakov DV, Suster S, LeBoit PE, et al. Mucinous carcinoma of the skin, primary, and secondary: a clinicopathologic study of 63 cases with emphasis on the morphologic spectrum of primary cutaneous forms: homologies with mucinous lesions in the breast. Am J Surg Pathol 2005; 29: 764-82.

2. Qureshi HS, Salama ME, Chitale D, et al. Primary cutaneous mucinous carcinoma: presence of myoepithelial cells as a clue to the cutaneous origin. Am J Dermatopathol 2004; 26: 353-8.

3. Headington JT. Primary mucinous carcinoma of skin: histochemistry and electron microscopy. Cancer 1977; 39: 1055-63.

4. Wright JD, Font RL. Mucinous sweat gland adenocarcinoma of eyelid: a clinicopathologic study of 21 cases with histochemical and electron microscopic observations. Cancer 1979; 44: 1757-68.

5. Pilgrim JP, Kloss SG, Wolfish PS, Heng MC. Primary mucinous carcinoma of the skin with metastases to the lymph nodes. Am J Dermatopathol 1985; 7: 461-9.

6. Martinez S, Young S. Primary mucinous carcinoma of the skin. Internet J Oncol 2004; 2: 1-7.

7. Requena L, Kiryu H, Ackerman AB. Neoplasms with apocrine differentiation. Philadelphia: Lippincott Williams \& Wilkins, 1998; 907-47.
8. Saigal RK, Khanna SD, Chander J. Apocrine gland carcinoma in axilla. Indian J Dermatol Venereol 1971; 37: 177-80.

9. Aso K, Sato N. A case of primary mucinous carcinoma of the skin and trichofolliculoma developed in the same tissue. Jpn J Clin Dermatol 1989; 43: 117-20.

10. Wako M, Nishimaki K, Kawamura N, et al. Mucinous carcinoma of the skin with apocrine-type differentiation: immunohistochemical studies. Am J Dermatopathol 2003; 25: 66-70.

11. Lennox B, Pearse AG, Richards HG. Mucin-secreting tumours of the skin with special reference to the so-called mixed-salivary tumour of the skin and its relation to hidradenoma. J Pathol Bacteriol 1952; 64: 865-80.

12. Robson A, Lazar AJ, Ben Nagi J, et al. Primary cutaneous apocrine carcinoma: a clinico-pathologic analysis of 24 cases. Am J Surg Pathol 2008; 32: 682-90.

13. Helwig EB, Graham JH. Anogenital (extramammary) Paget's disease: a clinicopathological study. Cancer 1963; 16: 387-403.

14. Pascual JC, Perez-Ramos M, Devesa JP, Kutzner H, Requena L. Extramammary Paget's disease of the groin with underlying carcinoma and fatal outcome. Clin Exp Dermatol 2008; 33: 595-8.

15. Hurt MA, Hardarson S, Stadecker MJ, Santa Cruz DJ. Fibroepithelioma-like changes associated with anogenital epidermotropic mucinous carcinoma: fibroepitheliomatous Paget phenomenon. J Cutan Pathol 1992; 19: 134-41.

16. Taniyama K, Suzuki H, Yamada A, Tahara E. Extramammary Paget's disease with an underlying mucinous adenocarcinoma of the buttocks. Gan No Rinsho 1988; 34: 379-84.

17. Matin RN, Gibbon K, Rizvi H, Harwood CA, Cerio R. Cutaneous mucinous carcinoma arising in extramammary Paget disease of the perineum. Am J Dermatopathol 2011; 33: 705-9.

18. Bowling JC, Powles A, Nasiri N, Searle A, Bunker CB. Spontaneous regression of extramammary Paget's disease after excision of primary apocrine carcinoma, in an immunosuppressed patient. Br J Dermatol 2005; 153: 676-7.

19. Hernandez JM, Copeland EM 3rd. Infiltrating apocrine adenocarcinoma with extramammary pagetoid spread. Am Surg 2007; 73: 307-9.

20. Seo SH, Shin DH, Sung HW. Apocrine carcinoma of the groin possibly associated with extramammary Paget's disease. Ann Dermatol 2011; 23: 519-22. 